\title{
触 New Disease Reports \\ First report of Fusarium commune and Fusarium redolens causing crown and root rot on tomato in Algeria
}

\author{
N. Hamini-Kadar ${ }^{1}$, V. Edel-Hermann ${ }^{2}$, N. Gautheron ${ }^{2}$ and C. Steinberg ${ }^{2}$

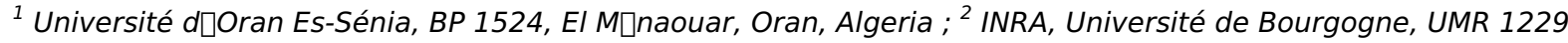 \\ Microbiologie du Sol et de I Environnement, CMSE, 17 rue Sully, BP 86510, 21065 Dijon, France \\ *E-mail: veronique.edel@dijon.inra.fr
}

Received: 12 Jul 2010. Published: 12 Jul 2010. Keywords: Fusarium oxysporum

Tomato (Solanum lycopersicum) is a common crop in Algeria. From 2006 to 2008, surveys were conducted in the tomato-growing areas of Algeria around Alger and Oran where diseased plants were found with yellow discolouration of the vascular tissue in stems, wilting of the lower leaves and crown and root rot. Fungal isolates with morphological characteristics similar to those of Fusarium oxysporum (Nelson et al., 1983) were collected from diseased plants and preserved in the MIAE collection (Microorganisms of Interest for Agriculture and Environment, INRA Dijon, France). Four isolates were identified using a two-locus molecular strategy recommended for Fusarium species, based on sequences of the internal transcribed spacer (ITS) region and the elongation factor EF-1a gene (Geiser et al., 2004). GenBank Accession Nos. are HM584894 to HM584901. A BLAST search identifiedisolates MIAE00129 and MIAE00131 as F. redolens and isolates MIAE00101 and MIAE00112 as $F$. commune based on $100 \%$ similarity with corresponding sequences. $F$. commune is a recently described species closely related to, but phylogenetically independent of $F$. oxysporum (Skovgaard et al., 2003).

The strains were evaluated for their pathogenicity on tomato. For each strain, ten 12-day-old seedlings of tomato cv. Monfavet 63.5 were inoculated by cutting the roots $7 \mathrm{~mm}$ below the crown and dipping the plants for five minutes in $5 \mathrm{ml}$ of a suspension of $10^{6}$ conidia/ml in minimal liquid medium (MLM). Plants were transplanted into perlite and the conidial suspension was poured onto the perlite around the crown. Ten non-inoculated plants were prepared using MLM instead of conidial suspension. Plants were incubated in a climatic chamber at $24^{\circ} \mathrm{C}$ during the day $(16 \mathrm{~h})$ and $20^{\circ} \mathrm{C}$ at night for four weeks. The percentages of plants affected (dead plants together with plants showing dark brown lesions on crown and roots) were $40 \%$ and $50 \%$ for $F$. redolens MIAE00129 and MIAE00131 respectively, and $80 \%$ and $0 \%$ for $F$. commune MIAE00101 and MIAE00112 respectively. No symptoms were observed on non-inoculated plants. The pathogenicity tests were performed again at a temperature of $28^{\circ} \mathrm{C}$ instead of $24^{\circ} \mathrm{C}$ during the day. The percentages of plants affected were $40 \%$ for MIAE00101 but $100 \%$ for the three other strains. To our knowledge, this is the first report of $F$. commune and F. redolens being pathogenic on tomato in Algeria.

\section{References}

Geiser DM, Jiménez-Gasco MD, Kang SC, Makalowska I, Veeraraghavan N, Ward TJ, Zhang N, Kuldau GA, O'Donnell K, 2004. FUSARIUM-ID v. 1.0: A DNA sequence database for identifying Fusarium. European Journal of Plant Pathology , 473-479.

[doi:10.1023/B:EJPP.0000032386.75915.a0]

Nelson PE, Toussoun TA, Marasas WFO, 1983. Fusarium species. An Illustrated Manual for Identification. University Park, PA, USA: The Pennsylvania State University Press.

Skovgaard K, Rosendahl S, O'Donnell K, Nirenberg HI, 2003. Fusarium commune is a new species identified by morphological and molecular phylogenetic data. Mycologia 95, 630-636. [doi:10.2307/3761939] 